OPEN ACCESS

Edited by:

Choong-Min Ryu,

Korea Research Institute

of Bioscience and Biotechnology,

South Korea

Reviewed by:

Richard Bostock,

University of California, Davis, USA

Lei Zhang,

North Carolina State University, USA

${ }^{*}$ Correspondence: Huiming Zhang,

Shanghai Center for Plant Stress

Biology, Shanghai Institutes for

Biological Sciences, Chinese Academy of Sciences, 3888 Chenhua

Road, Shanghai 201602, China hmzhang@sibs.ac.cn

Specialty section:

This article was submitted to

Plant Biotic Interactions,

a section of the journal

Frontiers in Plant Science

Received: 29 June 2015 Accepted: 10 September 2015 Published: 24 September 2015

Citation:

Liu X-M and Zhang H (2015)

The effects of bacterial volatile emissions on plant abiotic stress

tolerance.

Front. Plant Sci. 6:774.

doi: $10.3389 / \mathrm{fp} / \mathrm{s} .2015 .00774$

\section{The effects of bacterial volatile emissions on plant abiotic stress tolerance}

\author{
Xiao-Min Liu and Huiming Zhang* \\ Shanghai Center for Plant Stress Biology, Shanghai Institutes for Biological Sciences, Chinese Academy of Sciences, \\ Shanghai, China
}

Plant growth-promoting rhizobacteria (PGPR) are beneficial plant symbionts that have been successfully used in agriculture to increase seedling emergence, plant weight, crop yield, and disease resistance. Some PGPR strains release volatile organic compounds (VOCs) that can directly and/or indirectly mediate increases in plant biomass, disease resistance, and abiotic stress tolerance. This mini-review focuses on the enhancement of plant abiotic stress tolerance by bacterial VOCs. The review considers how PGPR VOCs induce tolerance to salinity and drought stress and also how they improve sulfur and iron nutrition in plants. The potential complexities in evaluating the effects of PGPR VOCs are also discussed. Keywords: plant growth-promoting rhizobacteria, volatile organic compounds, abiotic stress, salinity, drought,
sulfur nutrition, iron deficiency

\section{Introduction}

Plants live naturally with many microorganisms, and the nutrient-rich environment of the rhizosphere is especially conducive to interactions between microorganisms and plants. While many soil microorganisms have no observable effects on plants, others enhance or inhibit plant growth. Plant growth-promoting rhizobacteria (PGPR) are beneficial soil microorganisms that can stimulate plant growth or increase tolerance to stresses. Some PGPR have been applied in agriculture, resulting in increased seedling emergence, plant weight, crop yield, and disease resistance (Kloepper et al., 1980, 1991, 1999). PGPR promote plant growth by producing non-volatile substances, such as the hormones auxin and cytokinin, as well as 1-aminocyclopropane-1-carboxylate (ACC) deaminase, which reduces plant ethylene levels, and siderophores, which facilitate root uptake of metal nutrients (Loper and Schroth, 1986; MacDonald et al., 1986; Glick, 1999; Timmusk et al., 1999). In addition, certain PGPR promote plant growth by emitting volatile organic compounds (VOCs). Microbial VOC emission, which was reported in 2003 by Ryu et al., is now recognized as an important aspect of plant-microorganism interactions (Ryu et al., 2003; Wenke et al., 2010; Blom et al., 2011; Bitas et al., 2013; Farag et al., 2013). VOC emission is indeed a common property of a wide variety of soil microorganisms, although the identity and quantity of volatile compounds emitted vary among species (Effmert et al., 2012; Kanchiswamy et al., 2015).

Although PGPR VOCs do not contain any known plant growth hormones or siderophores (Farag et al., 2013; Kanchiswamy et al., 2015), VOC-mediated regulation of plant endogenous auxin homeostasis and of iron uptake by roots has been documented (Zhang et al., 2007, 2009;

Abbreviations: FIT1, Fe deficiency-induced transcription factor 1; FRO2, ferric reductase oxidase 2; HKT1, high affinity potassium transporter1; IRT1, iron regulated transporter 1; NR1 and 2, nitrate reductase 1 and 2; PEAMT, phosphoethanolamine N-methyltransferase; SOS, salt overly sensitive; VOCs, Volatile Organic Compounds. 


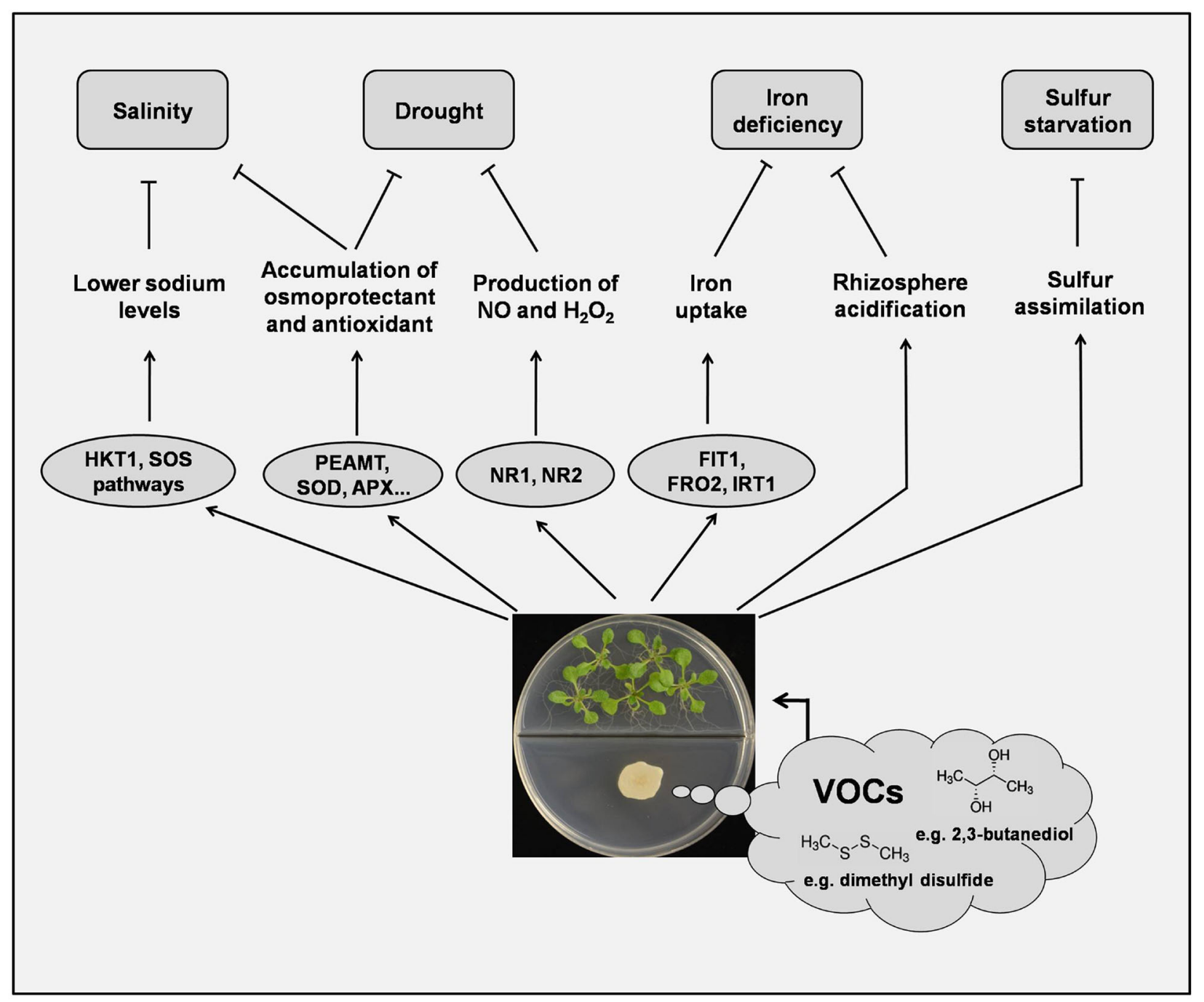

FIGURE 1 | The effects of microbial volatiles on plant abiotic stress tolerance. VOCs can modulate Arabidopsis sodium homeostasis via tissue-specific regulation of HKT1 and possibly also via the SOS pathway. Accumulation of $\mathrm{H}_{2} \mathrm{O}_{2}$ and nitric oxide is involved in the VOC-induced drought tolerance in plants. Accumulation of choline synthesized by VOC-induced PEAMT and other osmo-protectants may be a common mechanism for increasing osmotic protection in saltor drought-stressed plants. VOCs also trigger the expression of FIT1, FRO2, and IRT1 to facilitate iron uptake and plant growth. Under conditions of sulfur starvation, plants directly take up and assimilate the S-containing compounds (e.g., dimethyl disulfide) emitted from some PGPR. See text for details.

Farag et al., 2013). In addition to promoting plant growth, microbial VOCs may also induce disease resistance and abiotic stress tolerance, although the latter phenomenon has been studied only a few times. As a result of the increasing interests in VOCs in mediating plant-microorganism interactions, recent reviews have summarized the chemical nature of microbial VOCs, as well as the effects of microbial VOCs on plant biomass production and disease resistance (Wenke et al., 2010; Bailly and Weisskopf, 2012; Bitas et al., 2013; Farag et al., 2013; Garbeva et al., 2014; Audrain et al., 2015; Kanchiswamy et al., 2015). This mini-review focuses on the enhancement of plant abiotic stress tolerance by bacterial VOCs. In addition to providing an overview of PGPR VOC-induced tolerance to salinity and drought stress, we consider how PGPR VOCs improve sulfur and iron nutrition in plants (Figure 1). Finally, we discuss the potential complexities in evaluating the effects of PGPR VOCs.

\section{Increased Salt Tolerance}

Excessive sodium $\left(\mathrm{Na}^{+}\right)$creates both ionic and osmotic stresses for plant cells, leading to suppression of plant growth and reduction in crop yields (Zhu, 2001). Bacteria-induced salt tolerance in plants has been observed for several PGPR strains, among which Bacillus amyloliquefaciens GB03 (originally described as Bacillus subtilis GB03; Choi et al., 2014) displays VOC-mediated effects (Mayak et al., 2004; Barriuso et al., 2008; Zhang et al., 2008a). Salt-stressed Arabidopsis plants treated with GB03 VOCs showed greater biomass production and less $\mathrm{Na}^{+}$accumulation compared to salt-stressed plants without 
VOC treatment (Zhang et al., 2008a). Such VOC-induced stress tolerance was observed in wild-type (WT) plants but not in the hkt1 null mutant, suggesting a key role of HKT1 in mediating the salt stress tolerance triggered by GB03 VOCs. Arabidopsis HKT1 is a xylem parenchyma-expressed $\mathrm{Na}^{+}$transporter that is responsible for $\mathrm{Na}^{+}$exclusion from leaves by removing $\mathrm{Na}^{+}$from the xylem sap (Sunarpi et al., 2005; Horie et al., 2009; Møller et al., 2009). Under salinity stress, GB03 VOCs reduce $\mathrm{Na}^{+}$accumulation in Arabidopsis shoots, presumably by enhancing HKT1-dependent shoot-to-root $\mathrm{Na}^{+}$recirculation, because VOCs transcriptionally up-regulate $H K T 1$ in shoots and concomitantly down-regulate HKT1 in roots (Zhang et al., 2008a). While it remains unclear how GB03 VOCs regulate HKT1 transcription, the organ-specific patterns appear to be critical for VOC-induced salt tolerance as well as for auxin-mediated growth promotion (Zhang et al., 2007, 2008a).

GB03 VOCs decreased the $\mathrm{Na}^{+}$level in entire WT Arabidopsis plants by approximately $50 \%$, indicating either reduced $\mathrm{Na}^{+}$ uptake, enhanced $\mathrm{Na}^{+}$exudation, or both. Intriguingly, GB03 reduced plant $\mathrm{Na}^{+}$levels by only $15 \%$ in the Arabidopsis sos3 mutant (Zhang et al., 2008a). SOS3 is required for posttranscriptional activation of the $\mathrm{H}^{+} / \mathrm{Na}^{+}$antiporter SOS1, which controls root $\mathrm{Na}^{+}$exudation and long-distance $\mathrm{Na}^{+}$transport in plants (Shi et al., 2000). Therefore, SOS3-dependent $\mathrm{Na}^{+}$ exudation is likely required, as a part of the integrated regulation of $\mathrm{Na}^{+}$homeostasis, for the decreased accumulation of $\mathrm{Na}^{+}$ in VOC-treated plants. In addition, GB03 VOCs also cause rhizosphere acidification (Zhang et al., 2009), thereby producing a proton gradient that could potentially facilitate the SOS1mediated export of $\mathrm{Na}^{+}$from roots.

In response to salinity, plants adjust their endogenous metabolism to cope with osmotic stress caused by the excessive accumulation of $\mathrm{Na}^{+}$. PGPR-induced salt tolerance was recently reported in soybean plants exposed to volatile emissions from Pseudomonas simiae strain AU; the emissions not only decreased root $\mathrm{Na}^{+}$levels but also increased the accumulation of proline, which protect cells from osmotic stress (Vaishnav et al., 2015).Consistent with induced systemic tolerance under salinity, plants treated with AU VOCs showed higher levels of the vegetative storage protein (VSP) and several other proteins that are known to help sustain plant growth under stress conditions (Vaishnav et al., 2015).

\section{Protection from Water Loss}

Dehydration is a common threat to plants experiencing osmotic stress caused by salinity, drought, or cold conditions. Elevated accumulation of osmo-protectants in plants under dehydration stress can increase cellular osmotic pressure to lower the free water potential of cells and thereby prevent water loss, and can also stabilize proteins and membrane structures (Yancey, 1994). Under osmotic stress, Arabidopsis exposed to GB03 VOCs accumulated higher levels of choline and glycine betaine than plants without VOC treatment (Zhang et al., 2010). Choline and glycine betaine are important osmo-protectants that confer dehydration tolerance in plants (Rhodes and Hanson, 1993). Consistent with the elevated osmo-protectant levels, plants treated with GB03 VOCs or directly inoculated with GB03 displayed enhanced tolerance to dehydration stress. PEAMT, an essential enzyme in the biosynthesis pathway of choline and glycine betaine (Nuccio et al., 1998; Mou et al., 2002), was suggested to play a key role in mediating VOC-induced plant tolerance to dehydration, because VOC treatment increased the level of PEAMT transcripts and because genetic dysfunction of PEAMT abolished VOCinduction of dehydration tolerance (Zhang et al., 2010).

GB03 VOCs contain 2,3-butanediol, which promotes plant growth and induces disease resistance (Ryu et al., 2003, 2004). In addition to being found in GB03 VOCs, 2,3-butanediol is also found in the VOCs of some other PGPR strains including Pseudomonas chlororaphis strain O6, a bacterium that can trigger induced systemic resistance in plants. Under drought conditions, Arabidopsis plants inoculated with $P$. chlororaphis $\mathrm{O} 6$ or exposed to 2,3-butanediol exhibited increased stress tolerance, which evidently resulted from increased stomatal closure and reduced water loss (Cho et al., 2008). The application of $P$. chlororaphis O6 or 2,3-butanediol to mutants defective in various hormone signaling pathways indicated that the induced drought tolerance is regulated by multiple classic hormones including salicylic acid (SA), jasmonic acid (JA), and ethylene. In addition, SA appears to play a primary role in the induced drought tolerance, because free SA levels significantly increased in plants treated with P. chlororaphis O6 or 2,3-butanediol (Cho et al., 2008). In a subsequent study, 2,3-butanediol was found to induce plant production of nitric oxide (NO) and hydrogen peroxide, while chemical perturbation of $\mathrm{NO}$ accumulation impaired 2,3butanediol-stimulated plant survival under drought stress; these results indicated an important role for $\mathrm{NO}$ signaling in the drought tolerance induced by 2,3-butanediol (Cho et al., 2013).

The phytohormone abscisic acid (ABA) is known to control plant stress responses under dehydration conditions. However, the enhanced osmo-protection of plants treated with GB03 VOCs appears to be unrelated to $\mathrm{ABA}$, or at least to $\mathrm{ABA}$ production, because osmotic stress caused ABA to increase to similar levels in plants with and without exposure to GB03 VOCs (Zhang et al., 2010). That ABA is not the reason for PGPR-induced plant drought tolerance is further supported by observations that PGPR-treated Arabidopsis and cucumber plants accumulated less ABA than control plants (Cho et al., 2008; Kang et al., 2014). An indirect involvement of ABA in such PGPR-triggered abioticstress tolerance cannot be completely ruled out, however, given the complex cross-talk among ABA, NO, SA, and hydrogen peroxide signaling pathways in plants (Denancé et al., 2013; León et al., 2014; Song et al., 2014). PGPR-induced drought tolerance can also be mediated through elevated antioxidant responses at the levels of enzyme activity and metabolite accumulation, as was observed in wheat inoculated with Bacillus safensis strain W10 and Ochrobactrum pseudogregnonense strain IP8 (Chakraborty et al., 2013). Enhanced proline accumulation and gene expression of ROS-scavenging enzymes were observed in PGPR-treated potato plants, which displayed increased tolerance to various abiotic stresses including salinity, drought, and heavy-metal toxicity (Gururani et al., 2013). It would be useful to determine whether volatile emissions enhance antioxidative processes in plants that are stressed by dehydration. Some PGPR strains, such as 
Pseudomonas aeruginosa strain $\mathrm{Pa}$, produce exopolysaccharides that enhance the ability of the bacteria to maintain soil moisture content and increase drought tolerance in plants (Naseem and Bano, 2014). Certain bacterial VOCs such as acetic acid can induce the formation of biofilms, which contain exopolysaccharides as major constituents (Chen et al., 2015). Thus, it is possible that certain PGPR VOCs may indirectly increase plant drought tolerance by mediating exopolysaccharide production.

\section{Enhancement of Sulfur Acquisition}

As an essential element in many primary metabolites such as the amino acids cysteine and methionine, the macronutrient sulfur (S) is critical for plant survival. Under S-deficient conditions, plants suffer from repression of photosynthesis and disruption of primary metabolism(Burke et al., 1986; Gilbert et al., 1997). While plants mainly acquire $\mathrm{S}$ through root uptake of $\mathrm{SO}_{4}{ }^{2-}$ from soil, plants can also assimilate $\mathrm{S}$ from $\mathrm{S}$-containing compounds in the air, including some volatile compounds that are emitted by soil microorganisms (Meldau et al., 2013). Dimethyl disulfide (DMDS) is an S-containing volatile compound commonly produced by many soil bacteria and fungi (Kanchiswamy et al., 2015). Emission of DMDS from Bacillus sp. strain B55, a natural symbiont of Nicotiana attenuata plants, rescued plant growth retardation caused by S deprivation (Meldau et al., 2013). The incorporation of bacteria-emitted $S$ into plant proteins was demonstrated by adding radio-labeled ${ }^{35} \mathrm{~S}$ to the bacterial growth medium. In addition to detecting DMDS, Meldau et al. (2013) also detected the S-containing compound S-methyl pentanethioate in Bacillus sp. B55 VOCs. The authors attributed most of the S nutrition provided by Bacillus sp. B55 VOCs to DMDS rather than to S-methyl pentanethioate for two reasons. First, DMDS was detected as a major component of the volatile emissions while $S$ methyl pentanethioate was present in only trace amounts. Second, synthetic DMDS was superior to the natural VOC blends in rescuing S-starvation phenotypes of $N$. attenuata plants (Meldau et al., 2013).

Sulfur in $\mathrm{SO}_{4}{ }^{2-}$ is in an oxidative state and thus requires an energy-consuming reduction process for biological assimilation (Takahashi et al., 2011). In contrast, sulfur in DMDS is in a chemically reduced state. Therefore, it appears that DMDS may not only provide $S$ to plants but may also help plants avoid expending energy on sulfate reduction. Consistent with this hypothesis, DMDS supplementation significantly decreased the expression of $S$ assimilation genes as well as methionine biosynthesis and recycling (Meldau et al., 2013). Like DMDS in Bacillus sp. B55 VOCs, other S-containing volatile compounds such as dimethyl sulfide and dimethyl trisulfide have been detected in high concentrations in other microbial VOC blends (Kanchiswamy et al., 2015). Whether these microbial VOCs may also enhance $\mathrm{S}$ assimilation by plants remains to be determined.

\section{Optimization of Iron Homeostasis}

The transition between ferrous iron $\left(\mathrm{Fe}^{2+}\right)$ and ferric iron $\left(\mathrm{Fe}^{3+}\right)$ generates a redox potential that is important for electron transfer reactions including photosynthesis. Deprivation of Fe severely impairs the photochemical capacity and is accompanied by leaf chlorosis. Graminaceous monocots produce siderophores that increase $\mathrm{Fe}^{3+}$ mobility in soil and directly uptake $\mathrm{Fe}^{3+}$ without reduction, while non-graminaceous monocots and dicots not only acidify the rhizosphere to increase $\mathrm{Fe}^{3+}$ mobility but also use plasma membrane ferric reductase to reduce $\mathrm{Fe}^{3+}$ and subsequently transport $\mathrm{Fe}^{2+}$ into roots (Curie and Briat, 2003). Augmented Fe uptake was observed in Arabidopsis exposed to GB03 VOCs, which do not contain any known siderophores (Farag et al., 2006; Zhang et al., 2009). Under Fe-sufficient growth conditions, plants treated with GB03 VOCs displayed typical Fedeficiency responses, including transcriptional up-regulation of the root $\mathrm{Fe}^{3+}$ reductase gene FRO2 and of the $\mathrm{Fe}^{2+}$ transporter gene IRT1, increases in FRO2 enzyme activity, and rhizosphere acidification (Zhang et al., 2009). As a result, Fe levels were elevated in VOC-treated plants, consistent with greater amounts of Fe-rich photosynthetic apparatus (Zhang et al., 2008b).

GB03 VOC-triggered gene induction of IRT1 and FRO2 requires the transcription factor FIT1, because VOC failed to induce IRT1 or FRO2 in the fit1 knockout mutant (Zhang et al., 2009). VOC treatment also failed to increase iron uptake or photosynthesis in the fit1 mutant. Still, it remains unknown how VOC-treated plants initiate the inducible iron-deficiency responses. One possibility is that a demand for more iron may result from VOC-induced leaf cell expansion (Zhang et al., 2007) and/or photosynthesis augmentation (Zhang et al., 2008b). Also unclear is the identity of the component(s) in GB03 VOCs that induces plant iron-deficiency responses. On the other hand, acid components such as diethyl acetic acid possibly account for the rhizosphere acidification that is directly caused by VOC exposure (Farag et al., 2006; Zhang et al., 2009).

\section{Potential Complexities of VOC Effects on Plants}

Although PGPR VOCs have been shown to benefit plants via direct growth promotion, induced resistance to biotic stress, and increased tolerance to abiotic stress (Bailly and Weisskopf, 2012; Bitas et al., 2013; Farag et al., 2013), most of the data concerning these beneficial effects have been obtained in artificial environments. Current studies with PGPR VOCs typically use Iplates (Figure 1), in which a central partition separates plants from bacteria but allows bacterial VOCs to diffuse throughout the plate. This experimental setup appears to favor perception of volatile compounds by leaves, but in natural environments, PGPR VOCs that diffuse through rhizosphere soil pores would mainly be perceived by roots. Therefore, information obtained using I-plates may not apply to natural situations. Another concern with the use of I-plates is that, in addition to releasing volatiles, soil microorganisms also secrete non-volatile compounds (Glick, 1999), which may be taken up by roots and interfere with plant responses to VOCs.

Another complexity in studying the effects of PGPR VOCs on plants is that VOCs from the same PGPR strain may have different effects on plant growth and stress tolerance depending on the nature of the growth medium and the population density 
of the bacterium (Blom et al., 2011). An increase in the bacterial population in the same space may alter VOC profiles and result in the production of new, toxic components or elevated proportions or quantities of pre-existing toxic components. Alternatively, the VOC component that is responsible for plant growth promotion may accumulate to such high levels that it adversely affects plant growth. Indole, for example, promoted plant growth when applied at low levels but killed plants when applied at high levels (Blom et al., 2011). Similarly, both growth promotion and growth inhibition have been observed for plants treated with DMDS (Kai et al., 2010; Meldau et al., 2013). A change in plant growth conditions may also cause beneficial PGPR VOCs to become inhibitory. GB03 VOCs induce expression of FIT1 and IRT1 genes, which enhance plant iron uptake and photosynthesis (Zhang et al., 2009). In addition to transporting iron, IRT1 transports other metal ions such as cadmium into roots (Nishida et al., 2011). It therefore seems possible that GB03 VOCs may aggravate cadmium toxicity in cadmium-stressed plants.

\section{Conclusion}

To date, research on abiotic stress tolerance induced by PGPR VOCs in plants has revealed some interesting phenotypes and initial insights into underlying mechanisms. Nonetheless, further

\section{References}

Audrain, B., Farag, M. A., Ryu, C. M., and Ghigo, J. M. (2015). Role of bacterial volatile compounds in bacterial biology. FEMS Microbiol. Rev. 39, 222-233. doi: $10.1093 /$ femsre/fuu013

Bailly, A., and Weisskopf, L. (2012). The modulating effect of bacterial volatiles on plant growth: current knowledge and future challenges. Plant Signal. Behav. 7, 79-85. doi: 10.4161/psb.7.1.18418

Barriuso, J., Solano, B. R., and Gutierrez Manero, F. J. (2008). Protection against pathogen and salt stress by four plant growth-promoting rhizobacteria isolated from Pinus sp. on Arabidopsis thaliana. Phytopathology 98, 666-672. doi: 10.1094/PHYTO-98-6-0666

Bitas, V., Kim, H. S., Bennett, J. W., and Kang, S. (2013). Sniffing on microbes: diverse roles of microbial volatile organic compounds in plant health. Mol. Plant Microbe Interact. 26, 835-843. doi: 10.1094/MPMI-10-12-0249-CR

Blom, D., Fabbri, C., Connor, E. C., Schiestl, F. P., Klauser, D. R., Boller, T., et al. (2011). Production of plant growth modulating volatiles is widespread among rhizosphere bacteria and strongly depends on culture conditions. Environ. Microbiol. 13, 3047-3058. doi: 10.1111/j.1462-2920.2011.02582.x

Burke, J. J., Holloway, P., and Dalling, M. J. (1986). The effect of sulfur deficiency on the organization and photosynthetic capability of wheat leaves. J. Plant Physiol. 125, 371-375. doi: 10.1016/S0176-1617(86)80160-2

Chakraborty, U., Chakraborty, B. N., Chakraborty, A. P., and Dey, P. L. (2013). Water stress amelioration and plant growth promotion in wheat plants by osmotic stress tolerant bacteria. World J. Microbiol. Biotechnol. 29, 789-803. doi: 10.1007/s11274-012-1234-8

Chen, Y., Gozzi, K., Yan, F., and Chai, Y. (2015). Acetic acid acts as a volatile signal to stimulate bacterial biofilm formation. MBio 6:e00392. doi: $10.1128 / \mathrm{mBio}$. 00392-15

Choi, S. K., Jeong, H., Kloepper, J. W., and Ryu, C. M. (2014). Genome sequence of Bacillus amyloliquefaciens GB03, an active ingredient of the first commercial biological control product. Genome Announc. 2, e01092-14. doi: 10.1128/genomeA.01092-14

Cho, S. M., Kang, B. R., Han, S. H., Anderson, A. J., Park, J. Y., Lee, Y. H., et al. (2008). 2R,3R-butanediol, a bacterial volatile produced by Pseudomonas chlororaphis $\mathrm{O} 6$, is involved in induction of systemic tolerance to drought in Arabidopsis thaliana. Mol. Plant Microbe Interact. 21, 1067-1075. doi: 10.1094/MPMI-21-8-1067 insights into in planta molecular mechanisms are needed, especially regarding how VOC signals are perceived by plants and how plants assimilate certain VOC components as metabolites. Future research should also consider the possibility that PGPR VOCs have developed as a consequence of co-evolution. The survival of soil microorganisms is largely dependent on the growth and productivity of the plant community. In addition to supplying leaf litter for decomposers, plants also release up to $30 \%$ of their photosynthetic output in the form of root exudates that attract and maintain fungal and bacterial colonies in the soil (Smith et al., 1993; Jones et al., 2003). Therefore, mutually beneficial effects including enhancement of plant abiotic stress tolerance by PGPR VOCs could have resulted from the co-evolution of PGPR with their plant symbionts. Researchers have increasingly recognized that microbial VOCs play important roles in mediating interand intra-species interactions. Continued research on PGPR VOCs should lead to improved protection of plants from abiotic stress and to a better understanding of the underlying molecular mechanisms.

\section{Acknowledgment}

Research in the HZ laboratory is funded by the Chinese Academy of Sciences.

Cho, S. M., Kim, Y. H., Anderson, A. J., and Kim, Y. C. (2013). Nitric oxide and hydrogen peroxide production are involved in systemic drought tolerance induced by 2R,3R-butanediol in Arabidopsis thaliana. Plant Pathol. J. 29, 427-434. doi: 10.5423/PPJ.OA.07.2013.0069

Curie, C., and Briat, J. F. (2003). Iron transport and signaling in plants. Annu. Rev. Plant Biol. 54, 183-206. doi: 10.1146/annurev.arplant.54.031902. 135018

Denancé, N., Sánchez-Vallet, A., Goffner, D., and Molina, A. (2013). Disease resistance or growth: the role of plant hormones in balancing immune responses and fitness costs. Front. Plant Sci. 4:155. doi: 10.3389/fpls.2013. 00155

Effmert, U., Kalderás, J., Warnke, R., and Piechulla, B. (2012). Volatile mediated interactions between bacteria and fungi in the soil. J. Chem. Ecol. 38, 665-703. doi: 10.1007/s10886-012-0135-5

Farag, M. A., Ryu, C. M., Sumner, L. W., and Paré, P. W. (2006). GC-MS SPME profiling of rhizobacterial volatiles reveals prospective inducers of growth promotion and induced systemic resistance in plants. Phytochemistry 67, 2262-2268. doi: 10.1016/j.phytochem.2006.07.021

Farag, M. A., Zhang, H., and Ryu, C. M. (2013). Dynamic chemical communication between plants and bacteria through airborne signals: induced resistance by bacterial volatiles. J. Chem. Ecol. 39, 1007-1018. doi: 10.1007/s10886-0130317-9

Garbeva, P., Hordijk, C., Gerards, S., and de Boer, W. (2014). Volatile-mediated interactions between phylogenetically different soil bacteria. Front. Microbiol. 5:289. doi: 10.3389/fmicb.2014.00289

Gilbert, S. M., Clarkson, D. T., Cambridge, M., Lambers, H., and Hawkesford, M. J. (1997). SO42- deprivation has an early effect on the content of ribulose1,5-bisphosphate carboxylase/oxygenase and photosynthesis in young leaves of wheat. Plant Physiol. 115, 1231-1239.

Glick, B. R. (1999). "Overview of Plant Growth-Promoting Bacteria," in Biochemical and Genetic Mechanisms Used by Plant Growth Promoting Bacteria, eds B. R. Glick, C. N. Patten, G. Holguin, and D. M. Penrose (London: Imperial college Press), 1-13.

Gururani, M. A., Upadhyaya, C. P., Baskar, V., Venkatesh, J., Nookaraju, A., and Park, S. W. (2013). Plant growth-promoting rhizobacteria enhance abiotic stress tolerance in Solanum tuberosum through inducing changes in the expression of ROS-scavenging enzymes and improved photosynthetic performance. J. Plant Growth Regul. 32, 245-258. doi: 10.1007/s00344-012-9292-6 
Horie, T., Hauser, F., and Schroeder, J. I. (2009). HKT transporter-mediated salinity resistance mechanisms in Arabidopsis and monocot crop plants. Trends Plant Sci. 14, 660-668. doi: 10.1016/j.tplants.2009.08.009

Jones, D. L., Dennis, P. G., Owen, A. G., and van Hees, P. A. W. (2003). Organic acid behavior in soils-misconceptions and knowledge gaps. Plant Soil 248, 31-41. doi: 10.1023/A:1022304332313

Kai, M., Crespo, E., Cristescu, S. M., Harren, F. J., Francke, W., and Piechulla, B. (2010). Serratia odorifera: analysis of volatile emission and biological impact of volatile compounds on Arabidopsis thaliana. Appl. Microbiol. Biotechnol. 88, 965-976. doi: 10.1007/s00253-010-2810-1

Kanchiswamy, C. N., Malnoy, M., and Maffei, M. E. (2015). Chemical diversity of microbial volatiles and their potential for plant growth and productivity. Front. Plant Sci. 6:151. doi: 10.3389/fpls.2015.00151

Kang, S. M., Khana, A. L., Waqasa, M., You, Y. H., Kim, J. H., Kim, J. G., et al. (2014). Plant growth-promoting rhizobacteria reduce adverse effects of salinity and osmotic stress by regulating phytohormones and antioxidants in Cucumis sativus. J. Plant Interact. 9, 673-682. doi: 10.1080/17429145.2014.894587

Kloepper, J. W., Leong, J., Teintze, M., and Schroth, M. N. (1980). Enhanced plant growth by siderophores produced by plant growth promoting rhizobacteria. Nature 286, 885-886. doi: 10.1038/286885a0

Kloepper, J. W., Rodriguez-Kabana, R., Zehnder, G. W., Murphy, J., Sikora, E., and Fernandez, C. (1999). Plant root-bacterial interactions in biological control of soilborne diseases and potential extension to systemic and foliar diseases. Aust. J. Plant Pathol. 28, 27-33. doi: 10.1071/AP99003

Kloepper, J. W., Zablotowicz, R. M., Tipping, E. M., and Lifshitz, R. (1991). "Plant growth promotion mediated by bacterial rhizosphere colonizers," in The Rhizosphere and Plant Growth, eds K. L. Keister, and P. B. Cregan (Dordrecht: Kluwer), 315-326.

León, J., Castillo, M. C., Coego, A., Lozano-Juste, J., and Mir, R. (2014). Diverse functional interactions between nitric oxide and abscisic acid in plant development and responses to stress. J. Exp. Bot. 65, 907-921. doi: $10.1093 /$ jxb/ert454

Loper, J. E., and Schroth, M. N. (1986). Influence of bacterial sources of indole-3acetic acid on root elongation of sugar beet. Phytopathology 76, 386-389. doi: 10.1094/Phyto-76-386

MacDonald, E. M. S., Powell, G. K., Regier, D. A., Glass, N. L., Roberto, F., Kosuge, T., et al. (1986). Secretion of zwatin, Ribosylzeatin, and ribosyl-1" ${ }^{\prime \prime}$-methylzeatin by Pseudomonas savastanoi, plasmid-coded cytokinin biosynthesis. Plant Physiol. 82, 742-747. doi: 10.1104/pp.82.3.742

Mayak, S., Tirosh, T., and Glick, B. R. (2004). Plant growth-promoting bacteria confer resistance in tomato plants to salt stress. Plant Physiol. Biochem. 42, 565-572. doi: 10.1016/j.plaphy.2004.05.009

Meldau, D. G., Meldau, S., Hoang, L. H., Underberg, S., Wunsche, H., and Baldwin, I. T. (2013). Dimethyl disulfide produced by the naturally associated bacterium Bacillus sp B55 promotes Nicotiana attenuata growth by enhancing sulfur nutrition. Plant Cell 25, 2731-2747. doi: 10.1105/tpc.113.114744

Møller, I. S., Gilliham, M., Jha, D., Mayo, G. M., Roy, S. J., Coates, J. C., et al. (2009). Shoot $\mathrm{Na}^{+}$exclusion and increased salinity tolerance engineered by cell typespecific alteration of $\mathrm{Na}^{+}$transport in Arabidopsis. Plant Cell 21, 2163-2178. doi: $10.1105 /$ tpc.108.064568

Mou, Z., Wang, X., Fu, Z., Dai, Y., Han, C., Ouyang, J., et al. (2002). Silencing of phosphoethanolamine $\mathrm{N}$-methyltransferase results in temperature-sensitive male sterility and salt hypersensitivity in Arabidopsis. Plant Cell 14, 2031-2043. doi: 10.1105/tpc.001701

Naseem, H., and Bano, A. (2014). Role of plant growth-promoting rhizobacteria and their exopolysaccharide in drought tolerance of maize. J. Plant Interact. 9, 689-701. doi: 10.1080/17429145.2014.902125

Nishida, S., Tsuzuki, C., Kato, A., Aisu, A., Yoshida, J., and Mizuno, T. (2011). AtIRT1, the primary iron uptake transporter in the root, mediates excess nickel accumulation in Arabidopsis thaliana. Plant Cell Physiol. 52, 1433-1442. doi: $10.1093 / \mathrm{pcp} / \mathrm{pcr} 089$

Nuccio, M. L., Russell, B. L., Nolte, K. D., Rathinasabapathi, B., Gage, D. A., and Hanson, A. D. (1998). The endogenous choline supply limits glycine betaine synthesis in transgenic tobacco expressing choline monooxygenase. Plant J. 16, 487-496. doi: 10.1046/j.1365-313x.1998.00316.x

Rhodes, D., and Hanson, A. D. (1993). Quaternary ammonium and tertiary sulfonium compounds in higher plants. Annu. Rev. Plant Physiol. Plant Mol. Biol. 44, 357-384. doi: 10.1146/annurev.pp.44.060193.002041
Ryu, C. M., Farag, M. A., Hu, C. H., Reddy, M. S., Kloepper, J. W., and Paré, P. W. (2004). Bacterial volatiles induce systemic resistance in Arabidopsis. Plant Physiol. 134, 1017-1026. doi: 10.1104/pp.103.026583

Ryu, C. M., Farag, M. A., Hu, C. H., Reddy, M. S., Wei, H. X., Paré, P. W., et al. (2003). Bacterial volatiles promote growth in Arabidopsis. Proc. Natl. Acad. Sci. U.S.A. 100, 4927-4932. doi: 10.1073/pnas.0730845100

Shi, H., Ishitani, M., Kim, C., and Zhu, J. K. (2000). The Arabidopsis thaliana salt tolerance gene SOS1 encodes a putative $\mathrm{Na}^{+} / \mathrm{H}^{+}$antiporter. Proc. Natl. Acad. Sci. U.S.A. 97, 6896-6901. doi: 10.1073/pnas.120170197

Smith, J. L., Papendick, R. L., Bezdkek, D. F., and Lynch, J. M. (1993). “Soil organic matter dynamics and crop residue management," in Soil Microbial Ec, ed. F. B. Melting (New York: Marcel Dekker), 65-94.

Song, Y., Miao, Y., and Song, C. P. (2014). Behind the scenes: the roles of reactive oxygen species in guard cells. New Phytol. 201, 1121-1140. doi: 10.1111/nph.12565

Sunarpi, Horie, T., Motoda, J., Kubo, M., Yang, H., Yoda, K., et al. (2005). Enhanced salt tolerance mediated by AtHKT1 transporter-induced $\mathrm{Na}$ unloading from xylem vessels to xylem parenchyma cells. Plant J. 44, 928-938. doi: 10.1111/j.1365-313X.2005.02595.x

Takahashi, H., Kopriva, S., Giordano, M., Saito, K., and Hell, R. (2011). Sulfur assimilation in photosynthetic organisms: molecular functions and regulations of transporters and assimilatory enzymes. Annu. Rev. Plant Biol. 62, 157-184. doi: 10.1146/annurev-arplant-042110-103921

Timmusk, S., Nicander, B., Granhall, U., and Tillberg, E. (1999). Cytokinin production by Paenibacillus polymyxa. Soil Biol. Biochem. 31, 1847-1852. doi: 10.1016/S0038-0717(99)00113-3

Vaishnav, A., Kumari, S., Jain, S., Varma, A., and Choudhary, D. K. (2015). Putative bacterial volatile-mediated growth in soybean (Glycine max L. Merrill) and expression of induced proteins under salt stress. J. Appl. Microbiol. 119, 539-551. doi: 10.1111/jam.12866

Wenke, K., Kai, M., and Piechulla, B. (2010). Belowground volatiles facilitate interactions between plant roots and soil organisms. Planta 231, 499-506. doi: 10.1007/s00425-009-1076-2

Yancey, P. H. (1994). "Compatible and counteracting solutes," in Cellular and Molecular Physiology of Cell Volume Regulation, ed. K. Strange (Boca Raton, FL: CRC Press), 81-109.

Zhang, H., Murzello, C., Kim, M. S., Xie, X., Jeter, R. M., Zak, J. C., et al. (2010). Choline and osmotic-stress tolerance induced in Arabidopsis by the soil microbe Bacillus subtilis (GB03). Mol. Plant Microbe. Interact. 23, 1097-1104. doi: 10.1094/MPMI-23-8-1097

Zhang, H., Kim, M. S., Sun, Y., Dowd, S. E., Shi, H., and Paré, P. W. (2008a). Soil bacteria confer plant salt tolerance by tissue-specific regulation of the sodium transporter HKT1. Mol. Plant Microbe Interact. 21, 737-744. doi: 10.1094/MPMI-21-6-0737

Zhang, H., Xie, X., Kim, M. S., Kornyeyev, D. A., Holaday, S., and Pare, P. W. (2008b). Soil bacteria augment Arabidopsis photosynthesis by decreasing glucose sensing and abscisic acid levels in planta. Plant J. 56, 264-273. doi: 10.1111/j.1365-313X.2008.03593.x

Zhang, H., Kim, M. S., Krishnamachari, V., Payton, P., Sun, Y., Grimson, M., et al. (2007). Rhizobacterial volatile emissions regulate auxin homeostasis and cell expansion in Arabidopsis. Planta 226, 839-851. doi: 10.1007/s00425-0070530-2

Zhang, H., Sun, Y., Xie, X., Kim, M. S., Dowd, S. E., and Paré, P. W. (2009). A soil bacteria regulates plant acquisition of iron via deficiency-inducible mechanisms. Plant J. 58, 568-577. doi: 10.1111/j.1365-313X.2009.03803.x

Zhu, J.-K. (2001). Cell signaling under salt, water and cold stresses. Curr. Opin. Plant Biol. 4, 401-406. doi: 10.1016/S1369-5266(00)00192-8

Conflict of Interest Statement: The authors declare that the research was conducted in the absence of any commercial or financial relationships that could be construed as a potential conflict of interest.

Copyright (C) 2015 Liu and Zhang. This is an open-access article distributed under the terms of the Creative Commons Attribution License (CC BY). The use, distribution or reproduction in other forums is permitted, provided the original author(s) or licensor are credited and that the original publication in this journal is cited, in accordance with accepted academic practice. No use, distribution or reproduction is permitted which does not comply with these terms. 